\title{
The collagen microstructural changes of rat menisci and tibiofemoral cartilages under the influence of mechanical loading: An in vitro wear test of whole joints
}

\author{
Haoliuyang Cai ${ }^{\mathrm{a}}$, Zhixiu Hao ${ }^{\mathrm{a}, \mathrm{b}, *}$, Liying Xiao ${ }^{\mathrm{a}}$, Chao Wan ${ }^{\mathrm{c}}$ and Lingying Tong ${ }^{\mathrm{a}, \mathrm{b}}$ \\ ${ }^{a}$ Department of Mechanical Engineering, Tsinghua University, Beijing, China \\ ${ }^{\mathrm{b}}$ State Key Laboratory of Tribology, Tsinghua University, Beijing, China \\ ${ }^{\mathrm{c}}$ Department of Engineering Mechanics, Tsinghua University, Beijing, China
}

\begin{abstract}
.
BACKGROUND: Knee osteoarthritis (OA) is suggested to be induced by multi-factors, and mechanical environment is regarded as a risky factor.

OBJECTIVE: To investigate the effect of isolated mechanical factor on cartilage.

METHODS: An active wear test system was designed to perform parameters-controlled in vitro wear tests on rat knee joints with specific load magnitude, flexion-extension angle, and movement frequency. Six hind limbs of 9-month-old male SpragueDawley rats, with an additional spring on the medial side, were worn by using the custom-designed apparatus. Researchers observed both the menisci and tibial cartilages of these hind limbs using multiphoton laser scanning microscopy to analyze the change of the collagen microstructure caused by wear.

RESULTS: Collagen microstructure of both the medial and lateral meniscus became disordered under cyclic load. Some tissues on the surface of the medial tibial cartilage were removed and the middle layer of the medial compartment displayed cracks. On the contrary, the lateral tibial cartilage was intact.

CONCLUSIONS: The results implied that cyclic load caused menisci microstructure disarrangement prior to tibial cartilage damage and the collagen structure of mid-layer tibial cartilage failed before that of the superficial layer under the kinematics adopted in the study.
\end{abstract}

Keywords: Cartilage, wear test, collagen microstructure, multiphoton microscopy

\section{Introduction}

Knee osteoarthritis $(\mathrm{OA})$ is a common geriatric disease, which not only causes pains and mobility problems, but also aggravates psychological and financial burdens on the patients. It is estimated that more than 2.5 billion people are bothered by knee OA [1]. In China, the prevalence of knee OA in the aged people over sixty is as high as $19.4 \%$ [2]. It is known that hyaline articular cartilage and meniscus

\footnotetext{
${ }^{*}$ Corresponding author: Zhixiu Hao, Department of Mechanical Engineering and State Key Laboratory of Tribology, Tsinghua University, Beijing, China. Tel.: +86 1062785522; E-mail: haozx@tsinghua.edu.cn.
}

0928-7329/17/\$35.00 (C) 2017 - IOS Press and the authors. All rights reserved

This article is published online with Open Access and distributed under the terms of the Creative Commons Attribution NonCommercial License (CC-BY-NC 4.0). 
play crucial roles in the lubrication of knee joints, and the articular cartilage is altered in all joints with OA [3].

OA is often considered as a degenerative joint disease whose direct cause and initial change are still unknown. Since the mechanical environment of the knee have influence on the initiation of knee OA [4, 5], investigation of the initial change of articular cartilage and meniscus without metabolic activity may be helpful to reveal the initiation and progression of cartilage failure solely affected by the mechanical factor.

Wear tests are commonly used to investigate different factors in cartilage degeneration, and have been conducted on different species by different test methods in previous researches. Traditional pin-on-disk wear tests were conducted to investigate different methodologies of quantifying cartilage wear [6], to measure the friction coefficient between wet articular cartilage surfaces [7], and to test potential chondroplasty biomaterials [8]. Such tests required the joints dissected and samples shaped, and the motion was usually reciprocation or rotation, thus the test conditions were significantly different from the real conditions. The in vivo tests [9-11], which could ensure the actual load and motion, were adopted to investigate the relationship between the load alteration and the degenerative changes in rabbit joints [9] as well as rat joints [10], and to study the changes of femoral cartilage in response to treadmill running [11]. However, the actual load and motion were hard to be identified and quantified, and the test process was affected by many uncontrollable factors caused by the living experimental animals. It will be helpful to conduct parameters-controlled in vitro wear tests on whole animal joints, under imitative physiological load and motion. In such wear test, the test environment is similar to physiological environment, and the load magnitude, flexion-extension angle, and movement frequency can be controlled to isolate risky factors of cartilage degeneration. By comparing different specimens under different mechanical loads, the direct causes of cartilage failure can be concluded.

In this paper, we designed a wear test device to conduct in vitro wear test on rats' whole knee joints under certain cyclic load. The loading condition was programme-controlled so that result of certain mechanical environment could be collected. After the in vitro wear test, the morphological changes of the collagen microstructure in menisci and cartilages were observed using a multiphoton laser scanning microscope. We hypothesized that the collagen microstructure would be differently damaged after the wear test.

\section{Materials and methods}

\subsection{Wear test device and test protocol}

A custom-designed wear test device (Fig. 1) was developed to simulate an in vivo physiological condition for rat knee joints. The load magnitude, flexion-extension angle, and movement frequency of the device could be controlled. An active pendulum system was used to achieve the flexion and extension of the knee joint. The proximal femur was fixed on a three-dimensional micro-motional stage by two screws. The micro-motional stage allowed the device to adapt to specimens of different sizes by adjusting the position of a screw, and the equilibrium knee flexion angle could be set to a certain value from $50^{\circ}$ to $90^{\circ}$. The distal tibia was fixed on the pendulum arm by the tibia holder with the axial movement freedom unrestricted. The pendulum arm was attached to a servo motor and its swing angle and frequency could be accurately controlled.

Certain force along the tibia axis was loaded on the tibia by two springs. One end of the spring was connected to the tibia holder and the other was connected to the slide block, which was drawn by four 


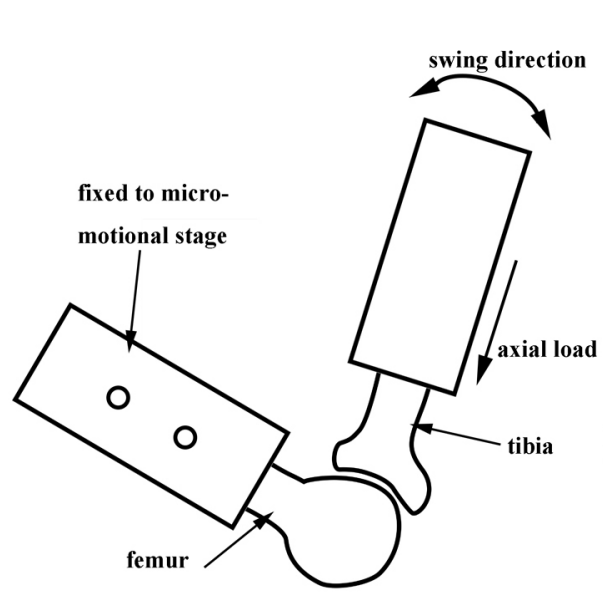

A

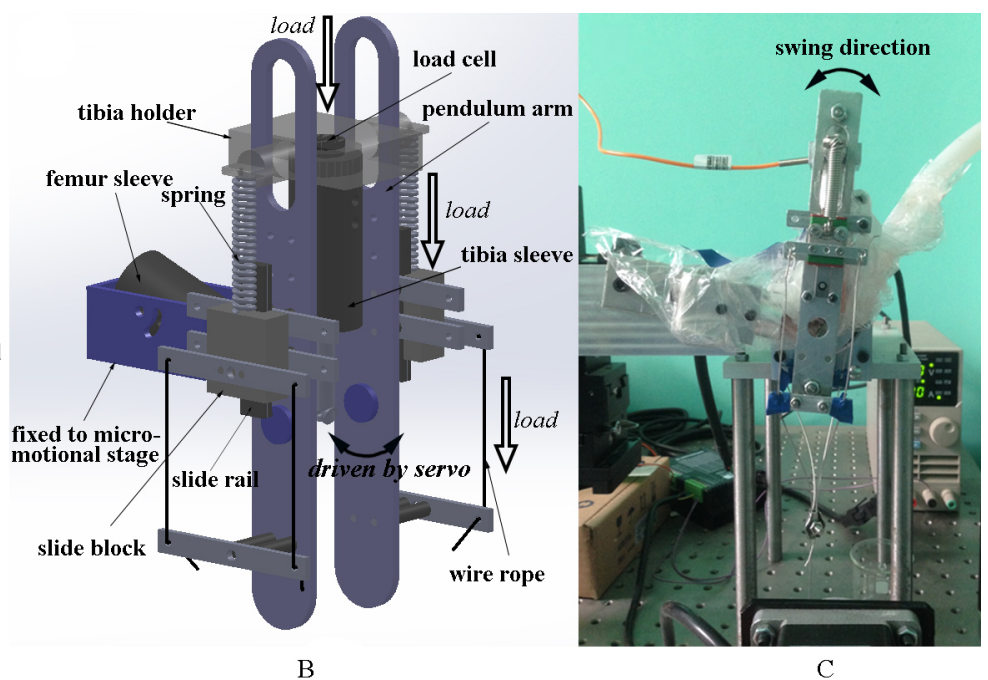

B

C

Fig. 1. Custom-designed wear test device. A) Schematic diagram of the wear test device. B) 3D model of the wear test device. a-tibia holder; b-load cell; c-pendulum arm; d-tibia sleeve; e-wire rope; f-slide rail; g-slide block; h-femur sleeve; i-spring. C) Running wear test device with a specimen and the semi-enclosed space.

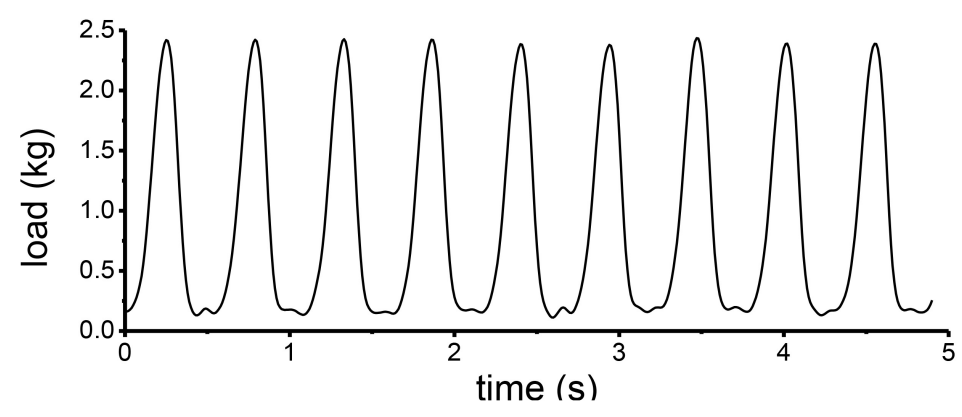

Fig. 2. Typical cyclic load in the wear test for one knee joint specimen from a 608-g rat.

wire ropes driven by a stepping motor to control the test load, alongside the pendulum arm (Fig. 1B). The actual test load was recorded by a load cell.

During the wear test, the specimens were wrapped in a plastic bag (Fig. 1C), which was full of atomized and heated $\left(35-37^{\circ} \mathrm{C}\right)$ PBS solution with antibiotics $(8 \mathrm{mg} / \mathrm{L}$ ampicillin, sodium salt and $32 \mathrm{mg} / \mathrm{L}$ streptomycin sulfate) in order to keep the joints under physiological conditions. The control group was also placed under the same environment as the experimental group to eliminate the influence of atomized PBS condition.

The loading spectrum (Fig. 2) of the wear test was determined according to the tibia load during normal locomotion. The equilibrium knee flexion angle was set to 70 degree [12] and the swing angle was set to 40 degree [13,14]. The frequency was set to $2 \mathrm{~Hz}$ (actual $1.86-1.90 \mathrm{~Hz}$, limited by the servo) [15]. The frequency of the cyclic load was synchronized with the swing movement, and the peak value of the cyclic load was four-bodyweight (BW) $[12,16]$. The total number of wear test cycles for the experimental group was 345000 , i.e. 51.5 hours, equal to the spontaneous locomotor activity of rat during 20 weeks $[17,18]$. 


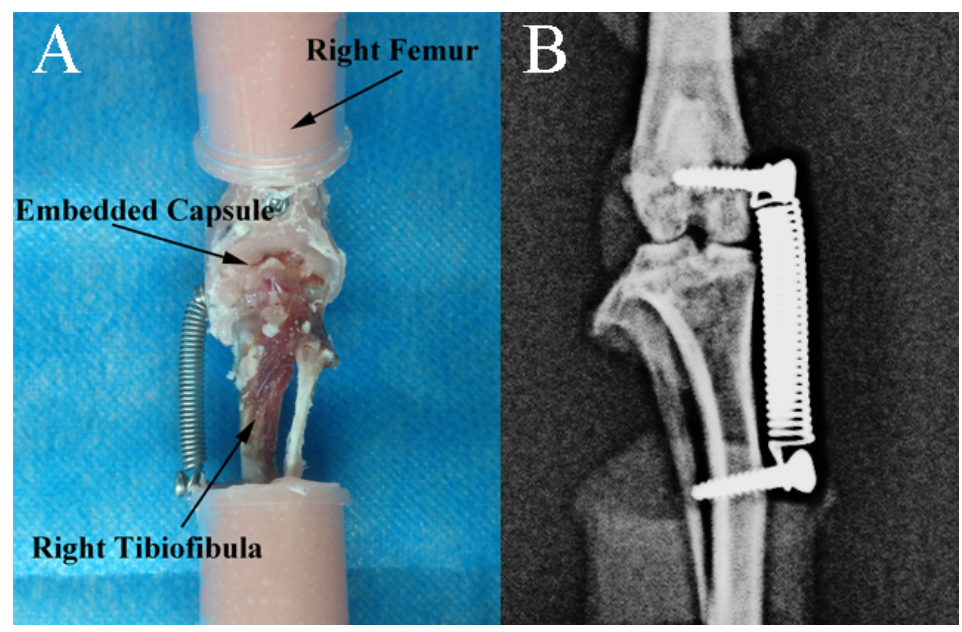

Fig. 3. Specimen used in the study. A) PMMA embedded rat femur, tibiofibula and capsule. B) X-ray image of the tension spring applied on the specimen to cause knee varus with a one-BW additional load.

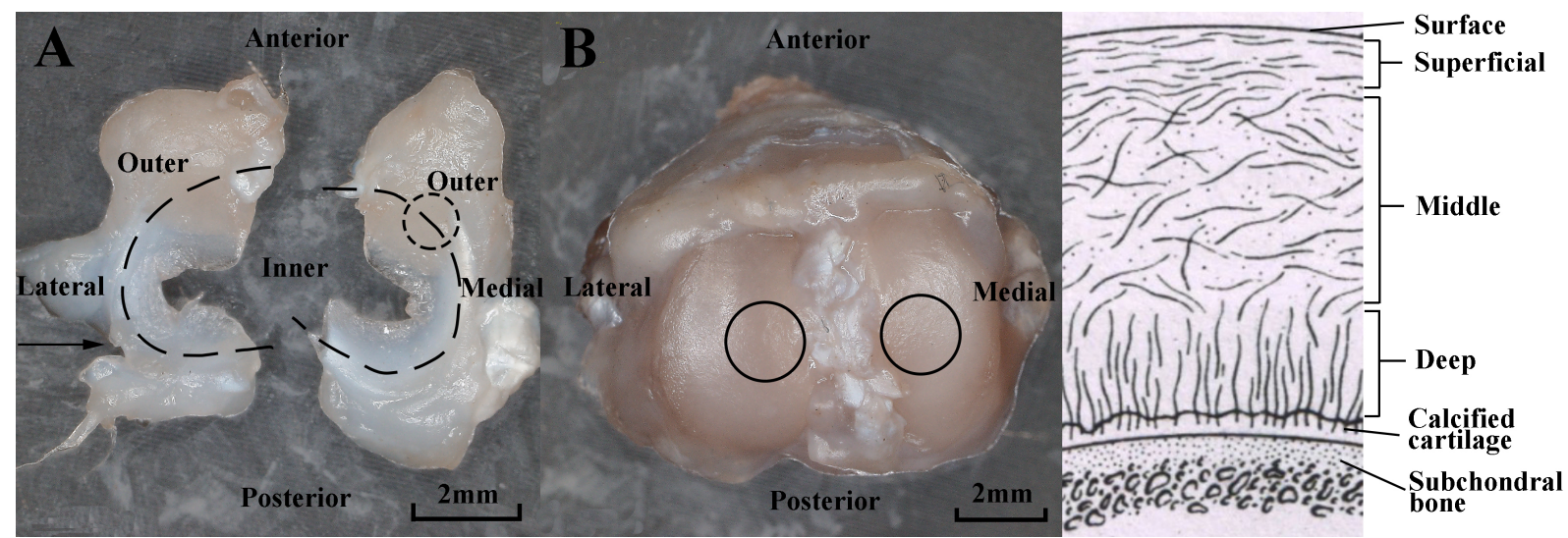

Fig. 4. Regional division of menisci and tibial cartilage. A) Optical image of menisci. Dash line circled area shows the typical observed region of menisci. B) Optical image of tibial plateaus. Solid line circled areas show the typical observed regions of tibial cartilage. C) Cross-sectional schematic of tibial cartilage showing a depth-dependent zone. Arrow in sub-figure A represents the slice direction.

\subsection{Specimen preparation}

All procedures were approved by the Institutional Animal Care and Use Committee of Tsinghua University. Six male Sprague-Dawley rats (9 month, $629.2 \pm 67.15 \mathrm{~g}$ ) were euthanized by a lethal dose of carbon dioxide, and both left and right hind limbs were resected from the animals. Intact capsules around the knee joints were reserved while soft tissues around the femur and tibiofibular stems were removed for a better fixation. All the right limbs were considered as the experimental group for wear tests while all the left limbs were selected as the control group. Both the proximal femur and distal tibia of the experimental group were embedded in polymethymethacrylic (PMMA) (Fig. 3A) and were fixed on the wear test device. The lower part of the capsule was resin embedded in PMMA to protect it from distal femoral fracture during the long time test, with free joint flexion and extension. In order to accelerate the 


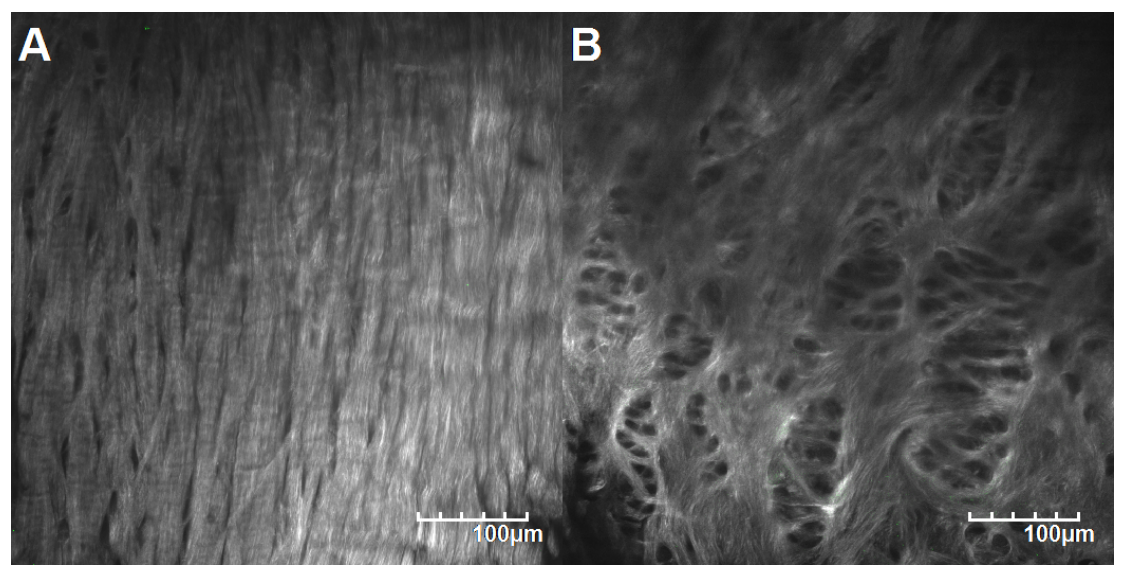

Fig. 5. Typical collagen microstructure of the outer region (A) and the inner region (B) of a healthy meniscus.

rate of wear in the medial part of cartilage, a one-BW additional load was applied on the medial tibial plateau of each experimental specimen by adding a stainless tension spring (Fig. 3B).

\subsection{Histological analysis}

Both of the menisci were resected, OCT-embedded, and sliced along the transverse plane (Fig. 4A). The central sites of the medial and lateral compartment surfaces of the tibial cartilage were observed to get the surface collagen microstructure and then transversely sliced to obtain the microstructure of different layers of the tibial cartilage (Fig. 4B and C). A multiphoton laser scanning microscope (FV1000MPE, Olympus, Japan) was used for observing the collagen microstructure. The excitation wavelength was set $850 \mathrm{~nm}$ [19], and two band pass filters (i.e., $420-460 \mathrm{~nm}$ and $495-540 \mathrm{~nm}$ ) were used to acquire the second harmonic generation (SHG) from collagen and the two-photon excited fluorescence(TPEF) from cells and elastin. A 25X, $1.05 \mathrm{NA}$ water immersion objective was used, and the image field was $508 \times$ $508 \mu \mathrm{m}^{2}$. Images from different parts of the meniscus and the tibial plateaus were collected to get the changes of collagen microstructure after the wear test.

The straightness of collagen fiber, which was defined as the ratio between fiber length and radial distance between starting point and ending point, was measured in some parts of the outer region of menisci to realize the preliminary quantification of collagen microstructure changes, using a previously described method [20]. After reconstructing the 2D images into 3D in Imaris (Bitplane, Switzerland), some distinguishable collagen fibers in a $200 \times 200 \times 10 \mu \mathrm{m}^{3}$ specified region were traced using the automated filament function of Imaris. Starting points and ending points were automatically created and artificially specified. The straightness of fiber was calculated by a built-in function, and according to the definition the value was always smaller than 1 .

\section{Results}

All specimens of the experimental group withstood the wear test without fracture because of the protective resin. After the wear test, the joints were intact and all uncut ligaments remained unbroken. With the joint dissected, the menisci were found intact, and no visible fibrillation or degeneration was observed on the tibia cartilage with India ink staining. 


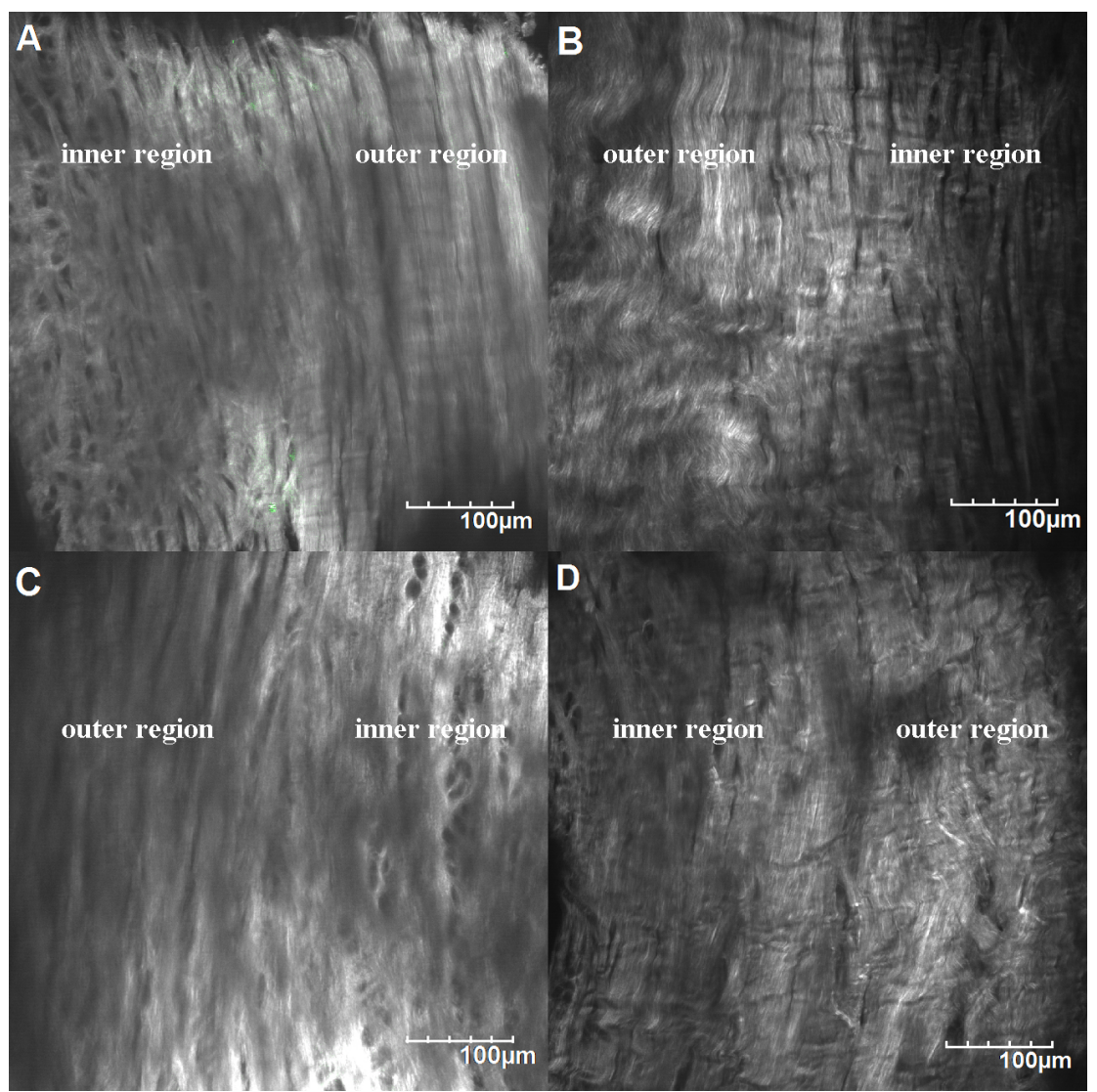

Fig. 6. Collagen microstructure of the menisci. A) Medial meniscus of the control group. B) Medial meniscus of the experimental group. C) Lateral meniscus of the control group. D) Lateral meniscus of the experimental group.

\subsection{Histological analysis of the menisci}

According to the SHG signals, the collagen microstructure was different in different regions of the mid-layer meniscus. As the unworn samples of the control group showed, the outer region of a normal meniscus displayed straight, compact and dense collagen bundles (Fig. 5A), while the collagen microstructure of the inner region was intertwined and sparse (Fig. 5B).

By scanning the field containing both the inner region and the outer region (Fig. 4A), the microstructure changes of the menisci were observed (Fig. 6). In many parts of the meniscus of experimental group, the collagen bundles of the outer region were differently out of order. Some of them became wavy and some of them were damaged. Less organized collagen were observed in both the medial and the lateral meniscus of the experimental group, which indicated that long-time, cyclic-load in vitro wear test would damage the collagen microstructure before visible fibrillation or degeneration displayed.

In the representative menisci showed in Fig. 7, seven distinguishable collagen fibers in the specified region were calculated and averaged. The average straightness of the medial meniscus of the control group was $0.998 \pm 0.001$, and the average straightness of the medial meniscus of the experimental group was $0.954 \pm 0.021$. Similarly, the average straightness of the lateral meniscus of the control group $(0.997 \pm 0.001)$ was higher than the experimental group $(0.972 \pm 0.008)$. 


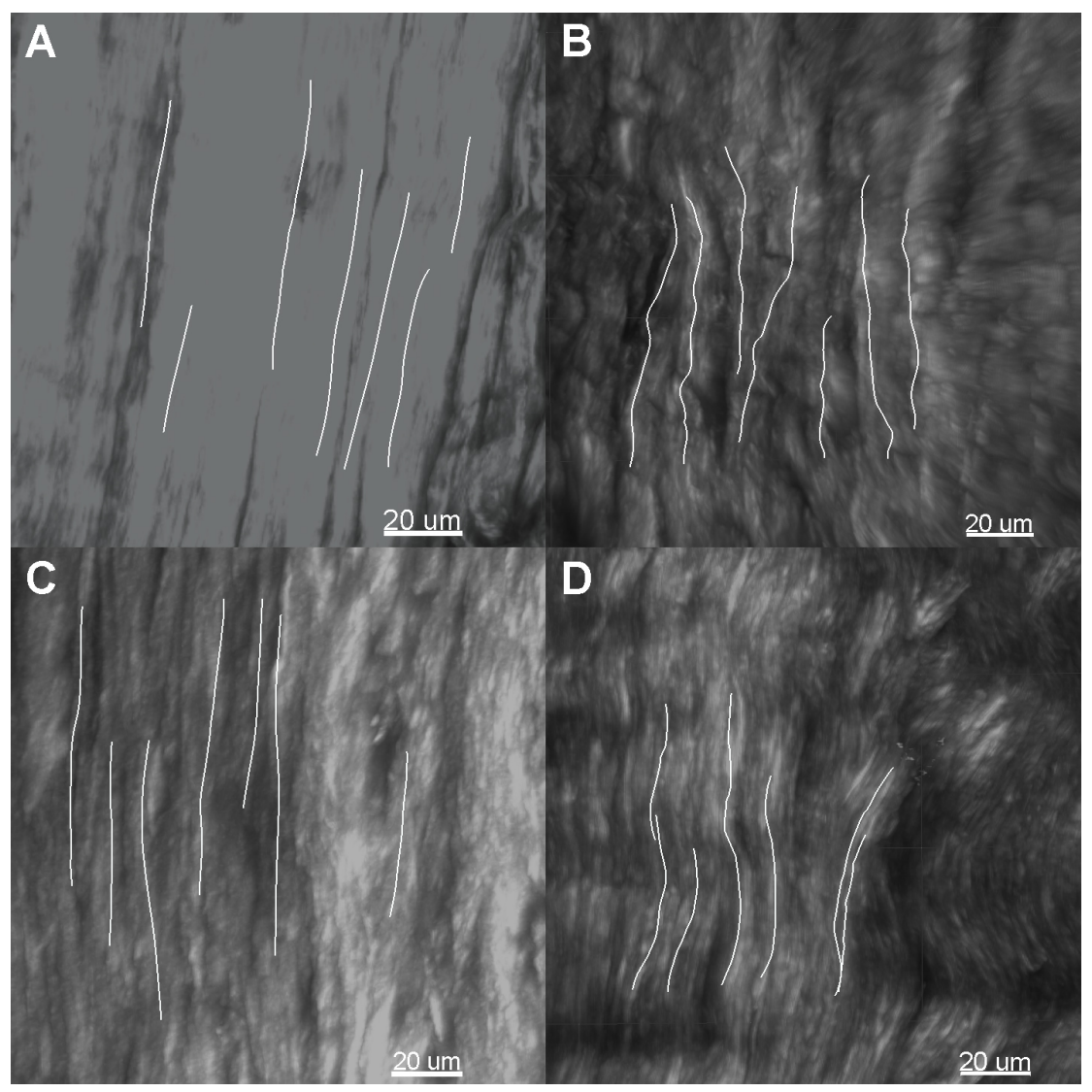

Fig. 7. Representative collagen fiber straightness measurement of the outer region of the menisci. A) Medial meniscus of the control group. B) Medial meniscus of the experimental group. C) Lateral meniscus of the control group. D) Lateral meniscus of the experimental group.

TPEF signals were faint in menisci, and even the gain value of TPEF signals was set very high, few signals could be detected. Information of elastin fibers could hardly be collected from such images. A previous study showed a similar conclusion that elastin fibers were not detected in rabbit menisci and the signal of elastin was absent [19].

\subsection{Histological analysis of the tibial cartilage}

Since the surface of tibial cartilage was uneven, the surface collagen microstructure commonly displayed along with the superficial layer microstructure of adjacent higher cartilage (Fig. 8). Collagen SHG signals showed that the surface of unworn tibial cartilage was completely covered by collagen, unlike the porous superficial layer. Micron-sized ostioles were observed on unworn cartilage surface (Fig. 8A and $\mathrm{C}$, circled area). The lateral compartment surface showed no obvious difference between the experimental group and the control group (Fig. 8D), while the surface of medial compartment became less collagen-covered and displayed no such ostiole as the control group (Fig. 8B).

The middle layer of the tibial cartilage showed different collagen microstructure from the surface (Fig. 9). The porous collagen structure of the lateral compartment displayed more sparsely, and the collagen took up less space than the medial compartment. For the cartilage of the experimental group, 


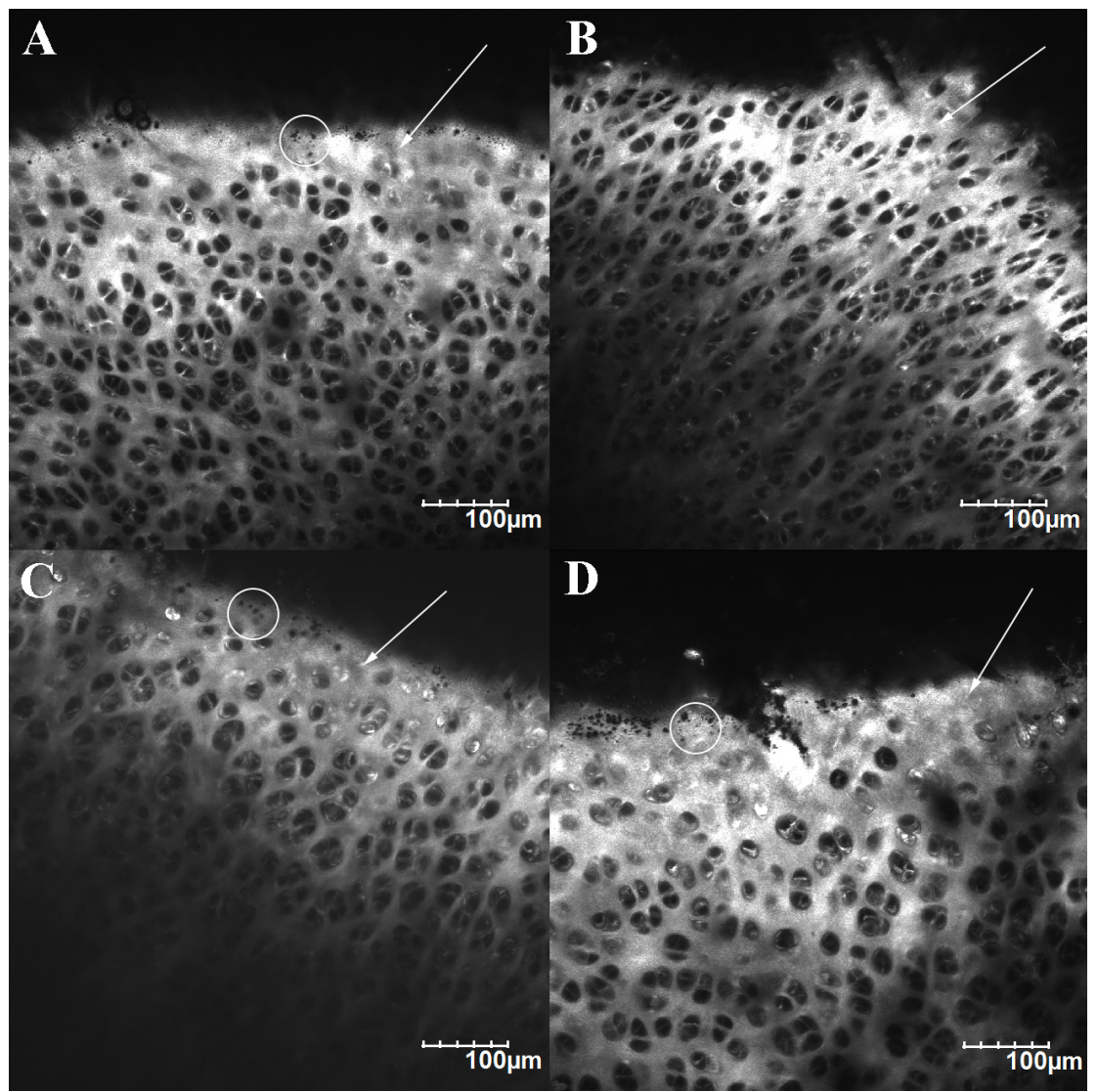

Fig. 8. Collagen microstructure of tibial cartilage surface and superficial layer. A) Medial compartment of the control group. B) Medial compartment of the experimental group. C) Lateral compartment of the control group. D) Lateral compartment of the experimental group. The arrows pointed out the surface on the focal plane. Circled areas show the micron-sized ostioles on the surface.

cracked collagen network was observed in the medial compartment (Fig. 9B), but the lateral compartment was intact as the control group. In the deep layer of the tibial cartilage, the SHG signal was intense around the TPEF-marked chondrocytes, and the network of collagen was not obvious. Cracks in such image could not be identified as the damage of cartilage caused by wear process.

The TPEF signals of the tibial cartilages were similar to the menisci, except that the TPEF signals from the cells were apparent. Yet no result of elastin fiber damage or cell damage could be concluded by the TPEF images. We mainly illustrated the SHG signals in this study because the TPFE signals showed less information.

\section{Discussion}

This study investigated the change of the collagen microstructure in meniscus and tibial cartilage after wear test under certain mechanical conditions. The less-ordered and cracked collagen of the experimental group verified the basic function of the wear test device.

After the wear test, no visible lesion or fibrillation was observed on the tibial cartilage or the menisci, but the alternation did exist through the results of multiphoton microscopy. Nevertheless, obvious fibril- 


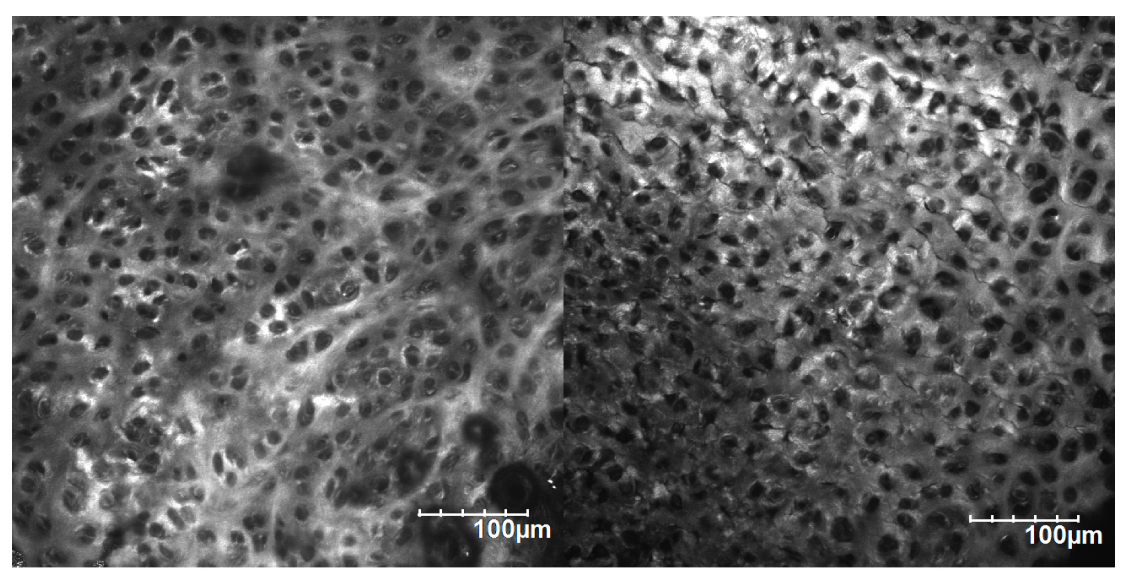

A

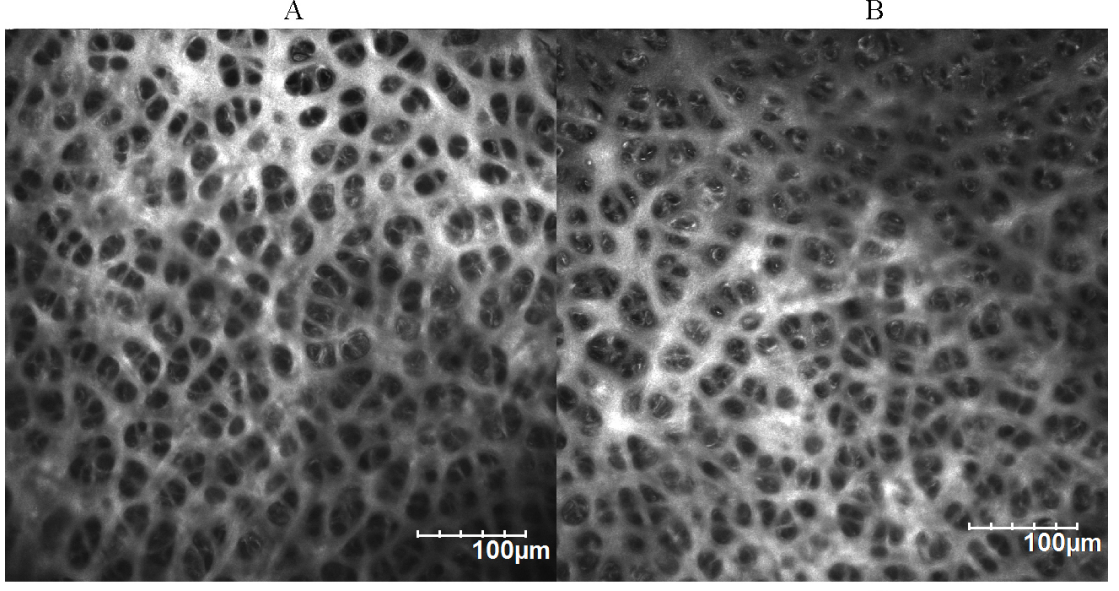

C

$\mathrm{D}$

Fig. 9. Collagen microstructure of the mid-layer cartilage. A) Medial compartment of the control group. B) Medial compartment of the experimental group. C) Lateral compartment of the control group. D) Lateral compartment of the experimental group.

lation was observed on the medial compartment of the tibial cartilage in a previous in vivo study [10], which we referred to when designing the load and the number of movements. The reason of such difference might be the kinematical difference, or the accelerated degeneration caused by the metabolic activities of living tissues under abnormal load. This study revealed that under the effect of similar mechanical environment, the change of the tibial cartilage and the meniscus started from inside structural failure, rather than surface lesions.

The histological analysis of the collagen microstructure revealed the change of collagen under abnormal mechanical environment. For the tested artificial varus knees, the collagen of the medial compartment's middle layer changed evidently as assumed and that of the surface showed recognizable difference, while no obvious difference was found in the lateral compartment, which proved the direct effect of mechanical infuence.

After the wear test, disarrangement of the collagen displayed mainly on the outer region of the meniscus, and the inner region seemed to be slightly affected, which implied that the ordered, compact outer region was the load bearing region in the meniscus. Because of the half-moon shape, in most part of the healthy meniscus, the collagen bundles were straight and compact. Unlike the tibial cartilage, both the medial and the lateral meniscus of the experimental groups showed difference from those of the corre- 
sponding control group. The change of the menisci might occur prior to the tibial cartilage under the kinematics adopted in this study.

One limitation of this study is that the changes of the collagen microstructure are still qualitative results. Although we measured the straightness of collagen fiber in some parts of the menisci in this study, other quantitative indicators such as collagen density and collagen fiber orientation are still needed to be studied to obtain creditable quantitative results of collagen microstructure changes. Furthermore, testing the mechanical properties of the tibial cartilages and menisci will be helpful to understand the change of knee tissues after wear test. Quantitative results will benefit a lot when studying the changes of cartilages under certain mechanical loading.

The study provided an effective way to carry out parameters-controlled wear test of rat's whole joints, under physiological conditions. The results of the multiphoton microscopy indicated that the differences were obvious. In this study, collagen microstructure of both the medial and lateral meniscus changed after the wear test, as well the medial compartment of tibial cartilage. More obvious collagen damages were observed on the surface and in the middle layer of medial tibial cartilage. Furthermore, the lateral meniscus changed before the lateral tibial cartilage. The collagen microstructural changes of the menisci and tibial cartilages implied the initiation of joint damage under adopted mechanical loading.

\section{Acknowledgments}

Financial supports from the project of Nature Science Foundation of China (51275267) are gratefully acknowledged.

\section{Conflict of interest}

None to report.

\section{References}

[1] Lim SS, Vos T, Flaxman AD, et al. A comparative risk assessment of burden of disease and injury attributable to 67 risk factors and risk factor clusters in 21 regions, 1990-2010: a systematic analysis for the Global Burden of Disease Study 2010. Lancet, 2012, 380(9859): 2224-2260.

[2] Xiang Y, Dai S. Prevalence of rheumatic diseases and disability in China. Rheumatology International, 2009, 29(5): 481-490.

[3] Loeser RF, Goldring SR, Scanzello CR, et al. Osteoarthritis: A disease of the joint as an organ. Arthritis \& Rheumatism, 2012, 64(6): 1697-1707.

[4] Andriacchi TP, Koo S, Scanlan SF. Gait Mechanics Influence Healthy Cartilage Morphology and Osteoarthritis of the Knee. Journal of Bone and Joint Surgery-American Volume, 2009, 91A: 95-101.

[5] Andriacchi TP, Mundermann A. The role of ambulatory mechanics in the initiation and progression of knee osteoarthritis. Curr Opin Rheumatol, 2006, 18(5): 514-518.

[6] Mcgann ME, Vahdati A, Wagner DR. Methods to assess in vitro wear of articular cartilage. Proceedings of the Institution of Mechanical Engineers Part H Journal of Engineering in Medicine, 2012, 226(8): 612-22.

[7] Shi L, Sikavitsas VI, Striolo A. Experimental friction coefficients for bovine cartilage measured with a pin-on-disk tribometer: testing configuration and lubricant effects. Annals of Biomedical Engineering, 2011, 39(1): 132-46.

[8] Northwood E, Fisher J. A multi-directional in vitro investigation into friction, damage and wear of innovative chondroplasty materials against articular cartilage. Clinical Biomechanics, 2007, 22(7): 834-842.

[9] Roemhildt ML, Beynnon BD, Gardner-Morse M, et al. Changes induced by chronic in vivo load alteration in the tibiofemoral joint of mature rabbits. J Orthop Res, 2012, 30(9): 1413-1422. 
[10] Roemhildt ML, Beynnon BD, Gauthier AE, et al. Chronic in vivo load alteration induces degenerative changes in the rat tibiofemoral joint. Osteoarthritis and Cartilage, 2013, 21(2): 346-357.

[11] Ni GX, Liu SY, Lei L, Li Z, Zhou YZ, Zhan LQ. Intensity-Dependent Effect of Treadmill Running on Knee Articular Cartilage in a Rat Model. Biomed Research International, 2013, 2013(2): 455-458.

[12] Drewniak EI, Jay GD, Fleming BC, et al. Cyclic loading increases friction and changes cartilage surface integrity in lubricin-mutant mouse knees. Arthritis \& Rheumatism, 2012, 64(2): 465-473.

[13] Gillis GB, Biewener AA. Hindlimb muscle function in relation to speed and gait: in vivo patterns of strain and activation in a hip and knee extensor of the rat (Rattus norvegicus). J Exp Biol, 2001, 204(Pt 15): 2717-2731.

[14] Canu MH, Garnier C, Lepoutre FX, Falempin M. A 3D analysis of hindlimb motion during treadmill locomotion in rats after a 14-day episode of simulated microgravity. Behavioural Brain Research, 2005, 157(2): 309-321.

[15] Clarke KA, Parker AJ. A quantitative study of normal locomotion in the rat. Physiol Behav, 1986, 38(3): 345-351.

[16] Wehner T, Wolfram U, Henzler T, et al. Internal forces and moments in the femur of the rat during gait. Journal of Biomechanics, 2010, 43(13): 2473-2479.

[17] Rodriguez VM, Carrizales L, Mendoza MS, et al. Effects of sodium arsenite exposure on development and behavior in the rat. Neurotoxicol Teratol, 2002, 24(6): 743-750.

[18] Bardullas U, Giordano M, Rodríguez VM. Chronic atrazine exposure causes disruption of the spontaneous locomotor activity and alters the striatal dopaminergic system of the male Sprague-Dawley rat. Neurotoxicology and Teratology, 2011, 33(2): 263-272.

[19] Levillain A, Boulocher C, Kaderli S, et al. Meniscal biomechanical alterations in an ACLT rabbit model of early osteoarthritis. Osteoarthritis and Cartilage, 2015, 23(7): 1186-1193.

[20] Hill MR, Duan X, Gibson GA, et al. A theoretical and non-destructive experimental approach for direct inclusion of measured collagen orientation and recruitment into mechanical models of the artery wall. Journal of Biomechanics, 2012, 45(5): 762-771. 